\title{
Helicobacter pylori can be related to carotid intima-media thickness, epicardial adipose tissue thickness and serum neutrophil gelatinase-associated lipocalin (NGAL) levels
}

\author{
Karadag $\mathrm{Z}^{1}$, Sehitoglu I², Cure $\mathrm{MC}^{3}$, Rakici $\mathrm{H}^{4}$, Ayvaz $\mathrm{MA}^{4}$, Bedir R ${ }^{5}$, Kizilkaya $\mathrm{B}^{6}$, \\ Şahin $\mathrm{OZ}^{7}$, Cure $\mathrm{E}^{8}$
}

Department of Internal Medicine, Camlica Erdem Hospital, Istanbul, Turkey. erkancure@yahoo.com

\begin{abstract}
BACKGROUND: Helicobacter pylori (HP) affects the cardiovascular system. Our aim in this study was to evaluate, whether an infection with HP causes subclinical atherosclerosis.

METHODS: We included 90 patients with dyspeptic symptoms in this study. The patients underwent an upper gastrointestinal endoscopy and biopsies were taken. The patients were grouped according to histopathologic examination, as HP infection negative $(n=21)$, HP infection positive $(+)(n=23)$, HP infection $(++)(n=22)$, HP infection $(+++),(n=24)$.

RESULTS: The neutrophilic gelatinase-associated lipocalin (NGAL) and high-sensitive C-reactive protein (hsCRP) levels and the carotid intima-media thickness (CIMT) and epicardial adipose tissue (EAT) thickness in the HP negative group were significantly lower than the NGAL $(p<0.001)$ and hs-CRP $(p<0.001)$ levels and the CIMT $(p<0.008)$ and EAT $(p<0.008)$ thickness in the HP $(+++)$ group. There was a strong correlation between the serum NGAL and hs-CRP levels, cIMT and EAT thickness.

CONCLUSION: HP-infection can lead to subclinical atherosclerosis via chronic inflammation. The higher the activity of HP infection, the higher the acceleration of atherosclerosis (Tab. 3, Fig. 2, Ref. 46). Text in PDF www.elis.sk. KEY WORDS: Helicobacter pylori, carotid intima-media thickness, epicardial adipose tissue thickness, neutrophilic gelatinase-associated lipocalin.
\end{abstract}

\section{Introduction}

Helicobacter pylori (HP) has a prevalence of $50 \%$ in general human population, in industrial countries this rate varies among middle-aged population between $20-50 \%$. HP is a coiled, flagellated microaerophilic gram-negative bacterium, which is found in the mucosa (1). Chronic HP-infection causes a severe damage in the gastric epithelium via chronic inflammation. It is known that HP infection can cause diseases of the gastrointestinal system, gastritis, duodenitis, and MALToma as well as cardiovascular, immunologic, hematologic pathologies and further extraintestinal diseases (2). Recently the close relationship between HP and cardiovascular pathologies was emphasized (3). The relationship

${ }^{1}$ Department of Cardiology, Versa Hospital, Nevsehir, Turkey, ${ }^{2}$ Department of Pathology, Private Practice, Rize, Turkey, ${ }^{3}$ Department of Biochemistry, Istanbul Laboratory, Istanbul, Turkey, ${ }^{4}$ Department of Gastroenterology, Recep Tayyip Erdogan University, Rize, Turkey, ${ }^{5}$ Department of Pathology, Recep Tayyip Erdogan University, Rize, Turkey, ${ }^{6}$ Department of Internal Medicine, Recep Tayyip Erdogan University, Rize, Turkey, ${ }^{7}$ Department of Nephrology, Medicana International Hospital, Istanbul, Turkey, and ${ }^{8}$ Department of Internal Medicine, Camlica Erdem Hospital, Istanbul, Turkey

Address for correspondence: E. Cure, MD, Department of Internal Medicine, Camlica Erdem Hastanesi, Uskudar, Istanbul, Turkey.

Phone: +90.850.2220494, Fax: +90.216.5226699 between the HP-associated secretion of proinflammatory cytokines on the one hand and atherosclerotic cardiac disease, on the other hand, has been reported (4).

The development of a HP infection-associated subclinical atherosclerosis has been announced (5). The measurement of carotid intima-media thickness (cIMT) is a useful and cheap marker in the detection of a subclinical atherosclerosis $(6,7)$. It is known that the epicardial adipose tissue (EAT) surrounding the coronary arteries is an active tissue and secretes proinflammatory cytokines (8). Increased EAT thickness is related to a rise of coronary heart disease and myocardial infarction (9). Lately it was indicated that the measurement of EAT thickness is an applicable and good marker in the detection of coronary heart disease (10).

Neutrophilic gelatinase-associated lipocalin (NGAL) (lipocalin-2), found in active neutrophils, is a protein, secreted in fat layer tissue, renal tubuli and epithelial tissues (11). NGAL as an acute phase protein is expressed in response to harmful stimuli in neutrophils, epithelial cells and renal tubular cells (12). Secretion of NGAL in gastric epithelium and the fact of an increase of NGAL secretion have been reported (13). In recent studies, the pathophysiologic importance of NGAL in cardiovascular diseases has been indicated $(14,15)$.

Because of HP infection, a chronic inflammation develops, which is a risk factor for cardiovascular diseases (16). In this 
study, we aimed to evaluate, if an HP infection causes subclinical atherosclerosis by measurement of cIMT and EAT thickness. In addition, we explored, whether HP-infection increases the secretion of NGAL and hs-CRP in subclinical atherosclerosis.

\section{Material and methods}

\section{Study population}

We included 90 patients attending the gastroenterology and internal medicine polyclinics with dyspeptic complaints in our study. Upper gastrointestinal endoscopy by a Gastroenterologist using Pentax-Endoscopes (EG-290 Kp, EG 2985 K) on a Pentax EPK 1000 Processor or Olympus-Endoscopes (CV-180) of the Evis Exera II series was performed in the patients. During endoscopy, 3 bioptic specimens in the antral region were taken, which were put in formaldehyde and sent to the department of pathology. The HP-frequency and chronic inflammation in the pathologic sampling were analyzed according to the Sydney classification semiquantitatively (17), unbeknown to the clinic and laboratory results. The examination was performed by 2 pathologists by the light optical microscopical technique with $400 \mathrm{x}$ magnification after dyeing with hematoxylin-eosin and Giemsa. Patients were grouped based on the histopathologic examination according to the Sydney classification as HP infection negative $(\mathrm{n}=21)$, HP infection $(+)(\mathrm{n}$ =23), HP infection $(++),(\mathrm{n}=22)$, HP infection $(+++)(\mathrm{n}=24)$.

Patients with an endoscopically detected active ulcer, bleeding gastrointestinal malignancy and active inflammation, intestinal metaplasia, dysplasia and carcinomatous finding were not included into the study. Patients with known malignancy, arterial hypertension, cardiac, renal or any kind of chronic disease, infection, hyperlipidemia and diabetes mellitus were excluded too. Patients smoking or with alcohol consumption, regular medication intake, taking of non-steroidal anti-inflammatory drugs, proton pump inhibitors or $\mathrm{H}_{2}$-receptor antagonists, anti-acid agents and previous use of an HP eradication regime within the last month did not participate in the study. The approval ethics committee was obtained as well as a signed patient informed consent.

\section{Echocardiographic evaluation}

The cardiologist made the measurement of the EAT and cIMT using a high-resolution 2-dimensional echocardiography (GEVingmed Ultrasound AS, Horten, Norway using a 2.5-3.5 MHz transducer) being clueless about the endoscopic, pathologic and laboratory results.

\section{EAT measurement}

The EAT was measured on the right ventricular outstanding wall in the parasternal long axis, using the aortic annulus as an anatomic reference according to the well-known method (8). In order to measure the EAT-thickness, we preferred the region above the right ventricle because of the fact, that the thickest layer of the EAT is right there. EAT was measured between the pericardial layers in front of the right ventricular outstanding wall at the end of the diastole as a peak (perpendicularly) as an echo free space. In order to measure the EAT more accurately and for a better vi- sualization each visualized picture was enlarged and the thickest point of EAT in each cycles was measured. We used the aortic annulus as an anatomic reference, in order to standardize the enlarged measuring axis. The measurement was made while the ultrasound waves pointed to the mid region of the right ventricular outstanding wall, and the aortic annulus was vertical.

\section{cIMT measurement}

The measurements on the right and left carotid arteries were performed with ultrasound in the supine position according to the previous method (6). With the common carotid arteries proximal and distal walls being parallel, the transducer was moved, so the diameter of the arteries lumen could be visualized in its maximal longitudinal extent. $1 \mathrm{~cm}$ proximal to the carotid bifurcation, the cIMT of the distant wall was assessed as the distance between the lumen and the intima and the distance between the media and adventitia. The cIMT was measured in an accurate frozen longitudinal picture and for a higher detail resolution, the picture was enlarged. The average value of the eight measurements on four with $1 \mathrm{~mm}$ distant adjacent localizations of the right and left carotid arteries was defined as cIMT.

\section{Biochemical analysis}

After a fasting period of 10-12 hours, the blood glucose was measured and the other biochemical parameters were analyzed with the Abbott Architect C16000 analyzer (Abbott Diagnostics, USA) using the photometric assay technique. The hemogram parameters were analyzed on the Abbott Cell-Dyn Ruby analyzer (Abbott Diagnostics, USA). Hs-CRP concentrations were measured using immunoturbidimetric assay with an Abbott Architect C 16000 analyzer (Abbott Diagnostic, USA). The cut-off for hsCRP was taken as $<0.5 \mathrm{mg} / \mathrm{dl}$.

\section{NGAL measurement}

The level of NGAL was measured using the enzyme-linked immunosorbent assay (ELISA) method. We used a commercially available human NGAL ELISA Kit (My Biosource, USA). The process for the application of the ELISA method was performed according to the instructions provided by the producer. The absorbance was measured at a wavelength of $450 \mu \mathrm{m}$. The levels of NGAL were presented as $\mathrm{ng} / \mathrm{mL}$, and the sensitivity of the NGAL assay was $<10 \mathrm{pg} / \mathrm{mL}$.

\section{Statistics analysis}

The results were presented as mean $\pm \mathrm{SD}$. The entire statistical tests were performed in SPSS version (version 18, IBM, Chicago, IL, USA). Because of the groups' number less than 30, nonparametric tests were used for the confrontation of the groups. For the confrontation of the groups, the Kruskal-Wallis test was applied. In cases of statistical significance in the Kruskal-Wallis test, the Bonferroni correction was made. Afterward, the groups were opposed to each other in binary items using the Mann-Whitney $U$ test. A p-value minor 0.008 was defined as significant. The correlation analysis was made with the Spearman rank test. The Stepwise linear regression analysis test was used. cIMT and EAT were 
Tab. 1. The results of cIMT, EAT thickness and laboratory parameters in HP infection.

\begin{tabular}{|c|c|c|c|c|}
\hline Parameters & $\begin{array}{c}\mathrm{HP}(-)(\mathrm{n}=21) \\
(\text { mean } \pm \mathrm{SD})\end{array}$ & $\begin{array}{c}\mathrm{HP}(+)(\mathrm{n}=23) \\
(\text { mean } \pm \mathrm{SD})\end{array}$ & $\begin{array}{c}\mathrm{HP}(++)(\mathrm{n}=22) \\
(\text { mean } \pm \mathrm{SD})\end{array}$ & $\begin{array}{c}\mathrm{HP}(+++)(\mathrm{n}=24) \\
\quad(\text { mean } \pm \mathrm{SD})\end{array}$ \\
\hline Age (years) & $52.0 \pm 7.9$ & $48.3 \pm 6.7$ & $50.2 \pm 8.0$ & $50.0 \pm 8.2$ \\
\hline Gender (M/F) (n) & $9 / 12$ & $13 / 10$ & 11/11 & $12 / 12$ \\
\hline BMI $\left(\mathrm{kg} / \mathrm{m}^{2}\right)$ & $27.8 \pm 1.5$ & $28.6 \pm 1.5$ & $28.5 \pm 1.7$ & $28.6 \pm 1.7$ \\
\hline Inflammation $(0-+++)$ & $0.1 \pm 0.3$ & $1.1 \pm 0.3 * *$ & $2.1 \pm 0.3 * * \dagger$ & $2.8 \pm 0.3^{* *+\tau}$ \\
\hline NGAL (ng/ml) & $173.1 \pm 23.1$ & $199.9 \pm 59.1$ & $214.7 \pm 51.6^{*}$ & $260.4 \pm 89.5 * * \dagger$ \\
\hline hs-CRP (mg/dl) & $0.10 \pm 0.1$ & $0.21 \pm 0.2$ & $0.28 \pm 0.2 *$ & $0.34 \pm 0.2 * \dagger \dagger$ \\
\hline $\operatorname{cIMT}(\mathrm{mm})$ & $0.67 \pm 0.08$ & $0.72 \pm 0.08$ & $0.77 \pm 0.09 *$ & $0.78 \pm 0.11^{*}$ \\
\hline FPG (mg/dl) & $98.8 \pm 9.2$ & $102.1 \pm 17.5$ & $102.8 \pm 21.4$ & $103.5 \pm 17.6$ \\
\hline BUN (mg/dl) & $34.9 \pm 11.9$ & $34.2 \pm 8.2$ & $31.4 \pm 8.3$ & $35.5 \pm 12.3$ \\
\hline Creatinine (mg/dl) & $0.81 \pm 0.12$ & $0.83 \pm 0.15$ & $0.79 \pm 0.07$ & $0.91 \pm 0.34$ \\
\hline AST (IU/l) & $25.6 \pm 14.2$ & $23.3 \pm 7.8$ & $23.4 \pm 9.9$ & $22.1 \pm 6.9$ \\
\hline ALT (IU/1) & $29.0 \pm 19.0$ & $25.8 \pm 16.0$ & $26.1 \pm 18.9$ & $22.1 \pm 8.1$ \\
\hline $\mathrm{WBC}\left(\mathrm{x} 10^{3} / 1\right)$ & $7.03 \pm 2.41$ & $8.16 \pm 2.48$ & $6.92 \pm 1.32$ & $7.51 \pm 2.75$ \\
\hline
\end{tabular}

Mann-Whitney U test with Bonferroni correction

ALT - alanine aminotransferase; AST - aspartate aminotransferase; BMI - body mass index; BUN - blood urea nitrogen; cIMT - carotid intima-media thickness; EAT epicardial adipose tissue; FPG - fasting plasma glucose; HP - Helicobacter pylori; hs-CRP - high sensitive C-reactive protein; NGAL - neutrophilic-gelatinase-associated lipocalin; WBC - white blood cell counts, ${ }^{*} \mathrm{p}<0.008, * * \mathrm{p}<0.001$ vs HP $(-), \uparrow \mathrm{p}<0.001, \dagger \dagger \mathrm{p}<0.008$ vs HP (+), 甲 p $<0.001$ vs HP (++)

used as dependent variables and for the detection of independent variables mostly influencing cIMT and EAT. A p minor 0.05 was declared as significant.

\section{Results}

There were no differences between age, BMI-score and gender. The NGAL and hs-CRP levels and the cIMT and EAT values of the HP negative group were significantly lower than the NGAL $(\mathrm{p}$ $<0.008)$ and hs-CRP $(\mathrm{p}<0.001)$ levels and the cIMT $(\mathrm{p}<0.008)$ and EAT $(\mathrm{p}<0.008)$ values of the HP $(++)$ group. Also, the NGAL and hs-CRP levels and cIMT and EAT values in the HP negative group were significantly lower than the NGAL $(\mathrm{p}<0.001)$ and hs-CRP $(p<0.001)$ and cIMT $(p<0.008)$ and EAT $(p<0.008)$ values of the HP $(+++)$ group. The NGAL level and EAT-value in the HP $(+)$ group was significantly lower than the NGAL level (p

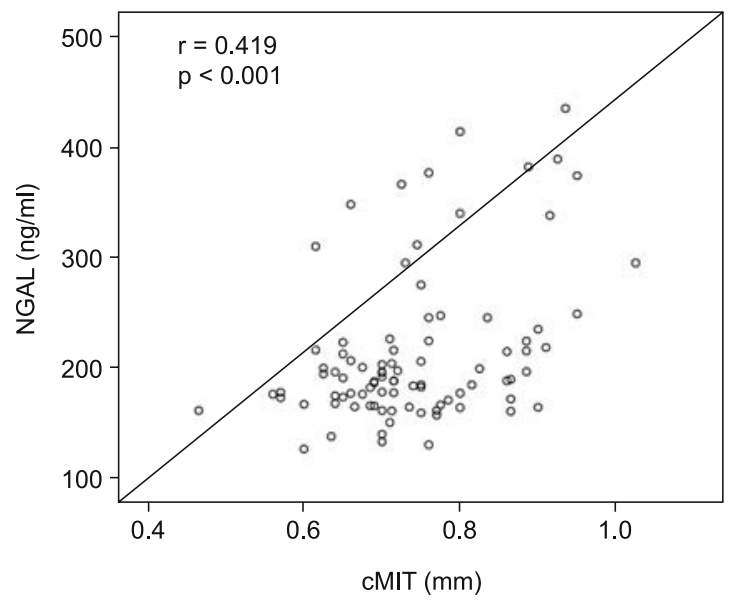

Fig. 1. Correlation between neutrophilic gelatinase-associated lipocalin and carotid intima-media thickness. NGAL, neutrophilic gelatinaseassociated lipocalin; cIMT, carotid intima-media thickness.
$<0.008)$ and EAT value $(\mathrm{p}<0.008)$ of the HP-group $(+++)$. The complete biochemical and social-demographic features of the HP $(-)$ and HP (+) group are listed in Table 1.

There was a positive relationship in the correlation analysis between a positive HP-status and gastric inflammation $(\mathrm{p}<0.001)$, $\operatorname{cIMT}(\mathrm{p}<0.001)$, EAT $(\mathrm{p}=0.003)$, NGAL $(\mathrm{p}<0.001)$ and hs-CRP $(p=0.001)$. Also, there was a positive relationship between cIMT and age $(p=0.005)$, EAT $(p=0.002)$, BMI $(r=0.639, p<0.001)$, gastric inflammation ( $p<0.001)$, NGAL $(p<0.001)$ (Fig. 1) and hs-CRP $(p<0.001)$. Furthermore, there was a positive relationship between EAT and gastric inflammation $(\mathrm{p}=0.022)$, BMI ( $p$ $<0.002)$, NGAL ( $p=0.007)$ (Fig. 2) and the hs-CRP ( $p=0.002)$. In Table 2 the results of the correlation analysis are shown. We also can see a positive relationship between NGAL and gastric inflammation $(\mathrm{r}=0.441, \mathrm{p}<0.001)$, BMI $(\mathrm{r}=0.230, \mathrm{p}=0.029)$, hs-CRP $(r=0.343, p=0.001)$ and BMI $(r=0.235, p=0.226)$.

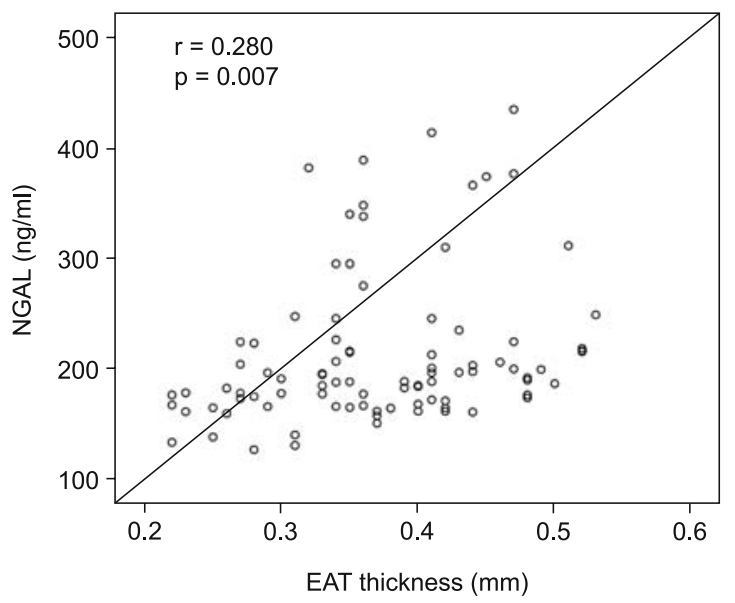

Fig. 2. Correlation between neutrophilic gelatinase-associated lipocalin and epicardial adipose tissue thickness. NGAL, neutrophilic gelatinaseassociated lipocalin; EAT, epicardial adipose tissue. 
Tab. 2. cIMT, EAT, HP and correlation analysis of other factors in patients.

\begin{tabular}{|c|c|c|c|c|c|c|}
\hline \multirow[b]{2}{*}{ Variable } & \multicolumn{2}{|c|}{ cIMT } & \multicolumn{2}{|c|}{ EAT } & \multicolumn{2}{|c|}{$\mathrm{HP}(+)$} \\
\hline & $\mathrm{r}$ & $\mathrm{p}$ & $\mathrm{r}$ & $\mathrm{p}$ & $\mathrm{r}$ & $\mathrm{p}$ \\
\hline Age & 0.293 & 0.005 & 0.120 & 0.258 & 0.057 & 0.595 \\
\hline BMI & 0.639 & 0.001 & 0.330 & 0.002 & 0.154 & 0.147 \\
\hline Inflammation & 0.438 & 0.001 & 0.235 & 0.022 & 0.951 & 0.001 \\
\hline cIMT & & & 0.329 & 0.002 & 0.418 & 0.001 \\
\hline EAT & 0.329 & 0.002 & & & 0.307 & 0.003 \\
\hline NGAL & 0.419 & 0.001 & 0.280 & 0.007 & 0.455 & 0.001 \\
\hline
\end{tabular}

Spearman rank test

BMI - body mass index; cIMT - carotid intima-media thickness; EAT - epicardial adipose tissue; HP - Helicobacter pylori; hs-CRP - high sensitive C-reactive protein NGAL - neutrophilic-gelatinase-associated lipocalin

Tab. 3. The results of dependent variables in stepwise linear regression analysis.

\begin{tabular}{lccc}
\hline Dependent variable & Independent variable & Beta & $\mathrm{p}$ \\
\hline cIMT & BMI & 0.503 & 0.001 \\
& Inflammation & 0.297 & 0.001 \\
& hs-CRP & 0.218 & 0.004 \\
& Age & 0.189 & 0.009 \\
\hline EAT & BMI & 0.289 & 0.004 \\
& HP positive & 0.263 & 0.009 \\
\hline
\end{tabular}

Stepwise linear regression analysis test

BMI - body mass index; cIMT - carotid intima-media thickness; EAT - epicardial adipose tissue; $\mathrm{HP}$ - Helicobacter pylori; hs-CRP - high sensitive C-reactive protein

In the stepwise regression analysis there was an independent relationship between cIMT and BMI (beta $[\beta]=0.503, \mathrm{p}<0.001)$, gastric inflammation $(\beta=0.297, \mathrm{p}=0.001)$, hs-CRP $(\beta=0.218$, $p=0.004)$ and age $(\beta=0.189, p=0.009)$. Also, there was an independent relation between EAT-thickness and BMI $(\beta=0.289$, $p=0.004)$ and HP-infection $(\beta=0.263, p=0.009)$. The complete results of the regression analysis are listed in Table 3.

\section{Discussion}

We evaluated, whether there is a relationship between HPinfection and its severity on the one hand and the serum NGAL and hs-CRP level on the other. We found that HP $(+)$ patients had a higher NGAL and hs-CRP-level in contrast to HP (-) patients and that there is a correlation between HP-infection and its severity and the level of NGAL and hs-CRP. It was shown that the cIMT and EAT values in patients with HP infection and gastritis were higher than in HP-negative patients and that with exceeding gastritis in $\mathrm{HP}(+)$ patients the cIMT and EAT values significantly increase. There was a strong correlation between serum NGAL and hs-CRP level and cIMT and EAT-value in the correlation analysis. In the regression analysis, there was an independent relation between cIMT and gastric inflammation, BMI, age, and hs-CRP. Further, there was an independent relationship between EAT and BMI and HP-status.

By infiltrating the gastric epithelium, HP leads to secretion of proinflammatory cytokines as tumor necrosis factor alpha (TNF- $\alpha$ ), interleukin (IL)-1 $\beta$ and IL-8 (18). The secretion of proinflammatory cytokines leads to a migration primarily of neutrophilic granulocytes, then lymphocytes, plasma cells and eosinophilic granulo- cytes to the target region (19). Thus, gastritis develops because of gastric epithelial damage (20). NGAL is an acute phase protein, secreted by neutrophilic granulocytes, epithelial cells, and tubular epithelial cells (12). In a case of an inflammation, hepatocytes, epithelial cells, macrophages and neutrophilic granulocytes, secrete NGAL (14). Alpizar-Alpizar et al reported a higher NGAL-secretion in gastric epithelial cells in HP-positive individuals compared to HP-negative individuals (13). It was demonstrated that the secretion of NGAL had an antibacterial effect on the IL-6 expression in some bacterial infections, like streptococcus pneumonia (21). Iron is an important element related to bacterial growth. By maintaining the iron homeostasis, NGAL plays an important role in inhibiting bacterial overgrowth (22). In our study, we found that with increasing HP-positivity the serum NGAL-level increased. In a case of an HP-infection, an increase of NGAL could be secondary to the inflammation or also to terminate the HP-infection.

The atherosclerotic process accelerates by increased cytokine secretion and more reactive oxygen radicals which cause more apoptosis (23). The increase cytokine expression leads to the expression of different lysosomal enzymes by increased production of neutrophilic granulocytes in the bone mark and by migration of neutrophilic granulocytes into the vessel walls (24). After the neutrophilic granulocytes have migrated into the vessel walls, the vascular endothelium is being damaged and atherosclerosis is accelerated (25). Especially young neutrophils amplify their NGAL-secretion as a response to the inflammation of the epithelial cells (12). On the other side, the secretion of NGAL leads to an upregulation of proinflammatory cytokines as IL-6 and IL-8 (26). Also proinflammatory cytokines cause an increase of NGAL level (27). As a result of the escalating inflammation, atherosclerosis increases even more.

It is reported, that the level of NGAL can be a predictive marker for an acute myocardial infarction, coronary heart disease, and cardiovascular events $(14,15)$. cIMT is a good and cheap indicator for subclinical atherosclerosis (23). A strong correlation has also been found between NGAL and cIMT $(28,29)$. Because of increased cytokine expression and inflammation in combination with HP-infection it is known that cardiac insufficiency and coronary heart disease develop $(30,31)$. Individuals infected with HP have been found to have higher levels of cIMT in comparison to HP-negative persons $(32,33)$. It is demonstrated that a decrease of paraoxonase-1 level in persons with HP-infection is associated 
with subclinical atherosclerosis $(34,35)$. In our study we found a strong correlation between NGAL and cIMT, as well as between NGAL and hs-CRP. We followed, that an increase of expression of NGAL could play an important role in the development of atherosclerosis. Nevertheless, we could not find an independent role between cIMT and NGAL in the regression analysis. Serum NGAL may not be a good and early predictive marker for the detection of subclinical atherosclerosis in patients with HP-infection.

EAT is a visceral fat tissue around the heart and the coronary arteries. This tissue actively secretes cytokines and others and its relationship to insulin resistance, metabolic syndrome, cardiac diseases are reported (36). With increasing EAT-thickness proinflammatory cytokines such as TNF- $\alpha$, IL- 6 , IL-8, angiotensin-II, plasma activator inhibitor and free fat acids increase and secretion of cardioprotective cytokines like adiponectin declines (8). A strong correlation between EAT and cIMT has been shown (37, $38)$. The EAT thickness was found to be $0-13.6 \mathrm{~mm}$ in the normal population in autopsy series (39). In our study, we found an increase of EAT with an increasing HP-positivity. Actually, EAT acts as a triglyceride storage for the myocardial tissue (40). It is known, that $\mathrm{HP}$ is related to non-alcoholic fatty liver disease (NAFLD) and HP-eradication decreases the fatty liver disease (41). The strong correlation between EAT and NAFLD is reported (42). The liver plays a role in EAT and in the increase of hepatic triglyceride accumulation. The gain of triglyceride accumulation and EAT play a role in the mechanism of the appearance of NAFLD. Therefore, HP-infection may amplify the EAT thickness by triglyceride accumulation. There is a positive strong correlation between HP and hs-CRP respectively NGAL. An increase of EAT-thickness secondary to HP-infection may be related to subclinical atherosclerosis and coronary artery disease, because with rising EAT thickness the secretion of cytokines in EAT increases. The increase of serum NGAL could induce the secretion of cytokines in EAT.

In our study, we determined the BMI as the strongest predictive parameter for cIMT and EAT. It is known, that the sensitivity for infections is increased in obese population (43). Zhang et al reported that the prevalence of HP-infection in overweight and obese patients is higher than in patients with normal weight (44). But other studies have shown an increase of HP-infection in obese patients $(45,46)$. In our study, the patients' BMI ranged between $25-30 \mathrm{~kg} / \mathrm{m}^{2}$. There were no obese patients. Therefore, we may not have found any correlation between HP and BMI. There was a strong correlation between cIMT, EAT, NGAL and hs-CRP levels in gastric inflammation. The increase of NGAL level in chronic gastric inflammation may increase the expression of proinflammatory cytokines and may accelerate the atherosclerotic process.

\section{Study Limitations}

The number of individuals in the study can be insufficient to represent the general population. Our study is only a pilot study, and further studies are needed on this topic. Obese subjects were not included in this study. Obese patients have higher level of inflammation than lean subjects. EAT thickness was evaluated only echocardiography, but it might be necessary to measure it by different methods such as magnetic resonance and computer tomography.

\section{Conclusion}

In HP positive individuals the cIMT and EAT-thickness and serum NGAL and the hs-CRP level were higher than in the healthy population. There was a strong correlation between serum NGAL and hs-CRP and cIMT and EAT thickness. HP-infection can lead to subclinical atherosclerosis by increasing the NGAL expression and inflammation. HP-infection can lead to an increase of EAT thickness. Also, in HP-positive patients EAT thickness can lead to cardiovascular diseases.

\section{References}

1. Suerbaum S, Michetti P. Helicobacter pylori infection. N Engl J Med 2002; 347 (15): 1175-1186.

2. Li Q, Lin $X, W u ~ Z$ et al. Immuno-histochemistry analysis of Helicobacter pylori antigen in renal biopsy specimens from patients with glomerulonephritis. Saudi J Kidney Dis Transpl 2013; 24 (4): 751-758.

3. Lin Y, Obata Y, Kikuchi S, Tamakoshi A, Iso H. JACC Study Group. Helicobacter pylori infection and risk of death from cardiovascular disease among the Japanese population: a Nested Case-Control Study within the JACC Study. J Atheroscler Thromb 2015; 22 (11): 1207-1213.

4. Rasmi Y, Raeisi S, Seyyed Mohammadzad MH. Association of inflammation and cytotoxin-associated gene a positive strains of helicobacter pylori in cardiac syndrome $\mathrm{x}$. Helicobacter 2012; 17 (2): 116-120.

5. Qu B, Wang H, Liu Y, Jia Y. Effects of H. pylori infection on carotid intima-media thickness, serum glucose, serum uric acid, liver and kidney function in subjects with chronic alcohol ingestion. Int J Cardiol 2015; 187: 470-471.

6. Cure MC, Tufekci A, Cure E et al. Low-density lipoprotein subfraction, carotid artery intima-media thickness, nitric oxide, and tumor necrosis factor alpha are associated with newly diagnosed ischemic stroke. Ann Indian Acad Neurol 2013; 16 (4): 498-503.

7. Icli A, Cure E, Cure MC et al. Endocan levels and subclinical atherosclerosis in patients with systemic lupus erythematosus. Angiology 2016; 67 (8): 749-755.

8. Cüre E, Ciçek Y, Cumhur Cüre M, Yüce S, Kirbaş A, Yilmaz A. The evaluation of relationship between adiponectin levels and epicardial adipose tissue thickness with low cardiac risk in Gilbert's syndrome: an observational study. Anadolu Kardiyol Derg 2013; 13 (8): 791-796.

9. Hajsadeghi F, Nabavi V, Bhandari A et al. Increased epicardial adipose tissue is associated with coronary artery disease and major adverse cardiovascular events. Atherosclerosis 2014; 237 (2): 486-489.

10. Sinha SK, Thakur R, Jha MJ et al. Epicardial adipose tissue thickness and its association with the presence and severity of coronary artery disease in clinical setting: A cross-sectional observational study. J Clin Med Res 2016; 8 (5): 410-419.

11. Giasson J, Li GH, Chen Y. Neutrophil gelatinase-associated lipocalin (NGAL) as a new biomarker for non--acute kidney injury (AKI) diseases. Inflamm Allergy Drug Targets 2011; 10 (4): 272-282.

12. Erturk A, Cure E, Parlak E, Cumhur Cure M, Baydur Sahin S, Yuce S. Clinical significance of neutrophil gelatinase-associated lipocalin in Crimean-Congo hemorrhagic Fever. Biomed Res Int 2015; 2015: 374010. 
13. Alpizar-Alpizar W, Laerum OD, Illemann M et al. Neutrophil gelatinase-associated lipocalin (NGAL/Lcn2) is upregulated in gastric mucosa infected with Helicobacter pylori. Virchows Arch 2009; 455 (3): 225-233.

14. Eilenberg W, Stojkovic S, Piechota-Polanczyk A et al. Neutrophil gelatinase-associated lipocalin (NGAL) is associated with symptomatic carotid atherosclerosis and drives pro-inflammatory state in vitro. Eur J Vasc Endovasc Surg 2016; 51 (5): 623-631.

15. Kirbis S, Gorenjak M, Sinkovic A. The role of urine neutrophil gelatinase--associated lipocalin (NGAL) in acute heart failure in patients with ST-elevation myocardial infarction. BMC Cardiovasc Disord 2015; 15: 49.

16. Kucukazman M, Yeniova O, Dal K, Yavuz B. Helicobacter pylori and cardiovascular disease. Eur Rev Med Pharmacol Sci 2015; 19 (19): 3731-3741.

17. Dixon MF, Genta RM, Yardley JH, Correa P. Classification and grading of gastritis. The updated Sydney System. International Workshop on the Histopathology of Gastritis, Houston 1994. Am J Surg Pathol 1996; 20 (10): 1161-1181.

18. Kivrak Salim D, Sahin M, Köksoy S, Adanir H, Süleymanlar I. Local Immune Response in Helicobacter pylori Infection. Medicine (Baltimore) 2016; 95 (20): e3713.

19. Figura N, Marano L, Moretti E, Ponzetto A. Helicobacter pylori infection and gastric carcinoma: Not all the strains and patients are alike. World J Gastrointest Oncol 2016; 8 (1): 40-54.

20. Bagheri N, Azadegan-Dehkordi F, Shirzad $M$ et al. Mucosal interleukin-21 mRNA expression level is high in patients with Helicobacter pylori and is associated with the severity of gastritis. Cent Eur J Immunol 2015; 40 (1): 61-67.

21. Warszawska JM, Gawish R, Sharif $\mathbf{O}$ et al. Lipocalin 2 deactivates macrophages and worsens pneumococcal pneumonia outcomes. J Clin Invest 2013; 123 (8): 3363-3372.

22. Abella V, Scotece M, Conde $\mathbf{J}$ et al. The potential of lipocalin-2/NGAL as biomarker for inflammatory and metabolic diseases. Biomarkers 2015; 20 (8): 565-571.

23. Icli A, Cure E, Cumhur Cure $M$ et al. Novel myokine: irisin may be an independent predictor for subclinic atherosclerosis in Behçet's disease. J Investig Med 2016; 64 (4): 875-881.

24. Amanzada A, Moriconi F, Mansuroglu T, Cameron S, Ramadori G, Malik IA. Induction of chemokines and cytokines before neutrophils and macrophage recruitment in different regions of rat liver after TAA administration. Lab Invest 2014; 94 (2): 235-247.

25. Singh RB, Mengi SA, Xu YJ, Arneja AS, Dhalla NS. Pathogenesis of atherosclerosis: A multifactorial process. Exp Clin Cardiol 2002; 7 (1): $40-53$.

26. Sousa MM, do Amaral JB, Guimarães A, Saraiva MJ. Up-regulation of the extracellular matrix remodeling genes, biglycan, neutrophil gelatinaseassociated lipocalin, and matrix metalloproteinase-9 in familial amyloid polyneuropathy. FASEB J 2005; 19 (1): 124-126.

27. Arena A, Stassi G, Iannello D et al. Both IL- $1 \beta$ and TNF- $\alpha$ regulate NGAL expression in polymorphonuclear granulocytes of chronic hemodialysis patients. Mediators Inflamm 2010; 2010: 613937.

28. Xiao Y, Xu A, Hui X et al. Circulating lipocalin-2 and retinol-binding protein 4 are associated with intima-media thickness and subclinical atherosclerosis in patients with type 2 diabetes. PLoS One 2013; 8 (6): e66607.

29. Gencer M, Gazi E, Hacivelioğlu $S$ et al. The relationship between subclinical cardiovascular disease and lipocalin-2 levels in women with PCOS. Eur J Obstet Gynecol Reprod Biol 2014; 181: 99-103.
30. Figura N, Palazzuoli A, Vaira D et al. Cross-sectional study: CagApositive Helicobacter pylori infection, acute coronary artery disease and systemic levels of B-type natriuretic peptide. J Clin Pathol 2014; 67 (3): 251-257.

31. Fouad FM, Mamer O, Sauriol F, Khayyal M, Lesimple A, Ruhenstroth-Bauer G. Cardiac heart disease in the era of sucrose polyester, Helicobacter pylori and Chlamydia pneumoniae. Med Hypotheses 2004; 62 (2): 257-267.

32. Basyigit S, Akbaş H, Süleymanlar I, Kemaloglu D, Koc S, Suleymanlar G. The assessment of carotid intima-media thickness, lipid profiles and oxidative stress markers in Helicobacter pylori-positive subjects. Turk J Gastroenterol 2012; 23 (6): 646-651.

33. Chen BF, Xu X, Deng Y et al. Relationship between Helicobacter pylori infection and serum interleukin-18 in patients with carotid atherosclerosis. Helicobacter 2013; 18 (2): 124-128.

34. Mete R, Oran M, Alpsoy $\mathbf{S}$ et al. Carotid intima-media thickness and serum paraoxonase-1 activity in patients with Helicobacter pylori. Eur Rev Med Pharmacol Sci 2013; 17 (21): 2884-2889.

35. Akbas HS, Basyigit S, Suleymanlar I et al. The assessment of carotid intima media thickness and serum paraoxonase-1 activity in Helicobacter pylori positive subjects. Lipids Health Dis 2010; 9: 92.

36. Aydin H, Toprak A, Deyneli O et al. Epicardial fat tissue thickness correlates with endothelial dysfunction and other cardiovascular risk factors in patients with metabolic syndrome. Metab Syndr Relat Disord 2010; 8 (3): $229-234$.

37. Altin C, Sade LE, Gezmis E et al. Assessment of subclinical atherosclerosis by carotid intima-media thickness and epicardial adipose tissue thickness in prediabetes. Angiology 2016; 67 (10): 961-969.

38. Elshorbagy HH, Fouda ER, Kamal NM, Bassiouny MM, Fathi WM. Evaluation of epicardial fat and carotid intima-media thickness in obese children. Iran J Pediatr 2016; 26 (1): e2968.

39. Schejbal V. Epicardial fatty tissue of the right ventricle--morphology, morphometry and functional significance. Pneumologie. 1989; 43 (9): 490-499.

40. Bertaso AG, Bertol D, Duncan BB, Foppa M. Epicardial fat: definition, measurements and systematic review of main outcomes. Arq Bras Cardiol 2013; 101 (1): e18-28.

41. Jamali R, Mofid A, Vahedi H, Farzaneh R, Dowlatshahi S. The effect of helicobacter pylori eradication on liver fat content in subjects with nonalcoholic Fatty liver disease: a randomized open-label clinical trial. Hepat Mon 2013; 13 (12): e14679.

42. Petta S, Argano C, Colomba D et al. Epicardial fat, cardiac geometry and cardiac function in patients with non-alcoholic fatty liver disease: association with the severity of liver disease. J Hepatol 2015; 62 (4): 928-933.

43. Falagas ME, Kompoti M. Obesity and infection. Lancet Infect Dis 2006; 6 (7): 438-446.

44. Zhang Y, Du T, Chen X, Yu X, Tu L, Zhang C. Association between Helicobacter pylori infection and overweight or obesity in a Chinese population. J Infect Dev Ctries 2015; 9 (9): 945-943.

45. Kyriazanos ID, Sfiniadakis I, Gizaris $\mathbf{V}$ et al. The incidence of Helicobacter pylori infection is not increased among obese young individuals in Greece. J Clin Gastroenterol 2002; 34 (5): 541-546.

46. Ioannou GN, Weiss NS, Kearney DJ. Is Helicobacter pylori seropositivity related to body mass index in the United States? Aliment Pharmacol Ther 2005; 21 (6): 765-272.

Received January 17, 2018. Accepted February 20, 2018. 